\title{
Excessive Daytime Sleepiness in Sleep Apnea: Any Role of Testosterone or Vitamin D?
}

\author{
Pavel ŠIARNIK ${ }^{\mathbf{1}}$, Matúš JURÍK ${ }^{\mathbf{1}}$, Miroslava HARDOŇOVÁ ${ }^{\mathbf{1}}$, Katarína KLOBUČNÍKOVÁ ${ }^{\mathbf{1}}$, \\ Jakub VEVERKA ${ }^{2}$, Pavol ŠURDA ${ }^{3}$, Peter TURČÁNI ${ }^{1}$, Branislav KOLLÁR ${ }^{1}$ \\ ${ }^{1}$ First Department of Neurology, Faculty of Medicine, Comenius University, Bratislava, Slovak \\ Republic, ${ }^{2}$ Department of Neurology, University Hospital Bratislava, Bratislava, Slovak Republic, \\ ${ }^{3}$ ENT Department, St. George's University Hospital, London, UK
}

Received June 2, 2020

Accepted August 25, 2020

Epub Ahead of Print September 9, 2020

\section{Summary}

Recent studies reported association of sleep-disordered breathing (SDB) with testosterone and vitamin D deficiency. Low testosterone and vitamin $D$ levels have been linked to fatigue and excessive daytime sleepiness (EDS). However, the impact of testosterone and vitamin D deficiency on EDS in subjects with SDB remains unknown. The aim of this study was to explore the predictors of EDS in habitual snorers. Role of testosterone, and vitamin $\mathrm{D}$ was studied in detail. We also looked for associations between testosterone, vitamin $\mathrm{D}$, and sleep-related indices. We prospectively enrolled 291 consecutive male patients with habitual snoring. Baseline clinical characteristics were recorded on admission. Standard overnight polysomnography was performed to detect SDB, and Epworth Sleepiness Scale (ESS) was used to assess EDS. Blood samples were obtained in a fasting condition in the morning after polysomnography to determine levels of testosterone and vitamin D. Respiratory disturbance index (RDI) (95\% CI: 1.004-1.024, $\mathrm{p}=0.005$ ) and the use of antihistamines (95\% CI: 1.083-11.901, $\mathrm{p}=0.037$ ) were the only independent variables significantly associated with EDS in binary logistic regression analysis. In linear multiple regression analysis, body mass index (BMI) (Beta $=-0.282$, $\mathrm{p}<0.001$ ) and oxygen desaturation index (Beta $=-0.150$, $p=0.043$ ) were the only independent variables significantly associated with testosterone levels, and BMI (Beta $=-0.142$, $\mathrm{p}=0.016$ ) was the only independent variable significantly associated with vitamin D. We failed to find any independent association of testosterone and vitamin $\mathrm{D}$ with subjectively rated EDS among habitual snorers. Our results suggest an independent association between the magnitude of nocturnal desaturation and testosterone levels.

\section{Key words}

Sleep-disordered breathing - Polysomnography - Excessive daytime sleepiness $\bullet$ Testosterone $\bullet$ Vitamin D

\section{Corresponding author}

P. Šiarnik, First Department of Neurology, Faculty of Medicine, Comenius University, Mickiewiczova 13, 813 69, Bratislava, Slovak Republic. E-mail: palo.siarnik@gmail.com

\section{Introduction}

Sleep-disordered breathing (SDB) is a group of frequent conditions that affects approximately $25 \%$ of adult males (Peppard et al. 2013). Typical symptoms and signs of SDB include snoring, apneas during sleep, as well as excessive daytime sleepiness (EDS) and fatigue (Cowie 2017, American Academy of Sleep Medicine 2014, Pagel 2006). Except of SDB, EDS is most commonly caused by sleep deprivation, medication or other medical, and psychiatric conditions. SDB is also associated with other sleep disorders including insomnia, restless legs syndrome (RLS), periodic limb movement disorder, and circadian rhythm disorders (American Academy of Sleep Medicine 2014).

Despite increasing data, the role of testosterone and vitamin D in sleep disorders remains controversial (Bercea et al. 2015, McCarty et al. 2014). Recent studies reported association of SDB with testosterone and vitamin D deficiency (Viana et al. 2017, Bouloukaki et al. 2020). Additionally, low testosterone and vitamin D levels have been linked to fatigue and daytime sleepiness 
(Hyde et al. 2010, McCarty et al. 2012). However, the impact of testosterone and vitamin D deficiency on EDS in subjects with SDB remains unknown.

The aim of this study was to explore the predictors of EDS in habitual snorers who were referred for sleep apnea evaluation in a sleep laboratory. Clinical characteristics, comorbidities and medication was considered, and the role of testosterone and vitamin D was studied in detail. We also looked for associations between testosterone, vitamin $\mathrm{D}$ and other sleep-related indices.

\section{Methods}

Between May 2016 and May 2017, we prospectively enrolled 291 consecutive adult male patients with habitual snoring who were examined at the sleep laboratory of the $1^{\text {st }}$ Department of Neurology, Comenius University Bratislava for sleep apnea evaluation. Subjects were excluded from a sleep study in a case of acute cardiac/respiratory comorbidity, or a case of acute exacerbation of chronic cardiac/respiratory comorbidity, end stage cancer, liver insufficiency, or if they refused to participate. Subjects were also excluded in a case of testosterone or vitamin D supplement therapy.

Clinical and demographic characteristics, including age, body mass index (BMI), past medical history and current medication, were obtained in all patients as a part of the baseline evaluation. Medical records of all patients were reviewed to search for medical conditions and medications that could contribute to EDS, including hypertension, diabetes mellitus, coronary artery disease, gastrointestinal disorders, renal insufficiency, liver dysfunction, hypothyroidism, cancer, parkinsonism, seizures, depression and the use of anticonvulsants, antidepressants, antiparkinsonian agents, alpha-adrenergic blocking agents, beta-adrenergic blocking agents, antihistamines and anxiolytics (Guilleminault and Brooks 2001, Pagel 2006, Pagel 2009). Subjects with absence of these conditions were considered as apparently healthy.

Overnight polysomnography (using Alice 6 device, Philips-Respironics, Netherlands) was performed in all patients. Standardized criteria were used to score sleep parameters and respiratory events. Apnea was defined as a cessation or a reduction of airflow of $\geq 90 \%$ for more than $10 \mathrm{~s}$. A reduction in airflow of $\geq 50 \%$ for more than $10 \mathrm{~s}$ associated with oxygen desaturation of $>3 \%$ was considered as a hypopnea. RERAs were estimated by flattening of the inspiratory airflow profile associated with an arousal, when airflow changes did not meet apnea or hypopnea criteria. A diagnosis of sleep apnea was defined as an apnea-hypopnea index (AHI) $\geq 5$. Respiratory disturbance index (RDI) was defined as the total number of apneas, hypopneas and respiratory effort related arousals (RERAs) per hour of sleep. Total sleep time (total sleep duration according to the polysomnography), arousal index (average number of arousals per hour sleep, AI), and oxygen desaturation index (average number of oxygen desaturation of $>3 \%$ per hour of sleep, ODI) were also recorded. Sleep stages (N1, N2, N3, REM) were scored according to the American Academy of Sleep Medicine sleep scoring rules (Iber et al. 2007).

The Epworth Sleepiness Scale (ESS) was used to assess EDS, and ESS score of 10 or more indicated the presence of EDS (Johns 1991). The minimum criteria defined by the International Restless Legs Syndrome Study Group were used to establish the diagnosis of the restless leg syndrome (RLS). To avoid false-positive diagnosis, the questionnaires were applied during an interview with a patient. Diagnosis of RLS was set when all four diagnostic criteria were fulfilled (an urge to move the legs, usually accompanied or caused by uncomfortable and unpleasant sensations in the legs; the urge to move or unpleasant sensations beginning or worsening during periods of rest or inactivity such as lying or sitting; the urge to move or unpleasant sensations partially or totally relieved by movement, such as walking or stretching; the urge to move or unpleasant sensations worse in the evening or night than during the day or only occurring in the evening or night) (Allen et al. 2003). Scorers were blinded to the baseline characteristics of the study population and to the results of other laboratory tests.

Blood tests were performed in the morning following polysomnography, and blood samples were obtained in a fasting condition within $1 \mathrm{~h}$ after polysomnography. Serum levels of vitamin D [25(OH)-D vitamin], and total testosterone were assessed. Testosterone levels were assessed by electrochemilumi-nescence immunoassay, and vitamin D levels were assessed by electrochemiluminescence immunoassay using Roche Cobas assay reagent (Roche Diagnostics, Mannheim, Germany). Testosterone levels were considered as decreased if they were $<9 \mathrm{nmol} / 1$ in 18-50-year-old males, or $<7 \mathrm{nmol} / \mathrm{l}$ in males older than 50 years. Hypovitaminosis D was defined as vitamin D $<20 \mu \mathrm{g} / \mathrm{l}$, and vitamin D insufficiency as vitamin D $<30 \mu \mathrm{g} / \mathrm{l}$. 
SPSS version 18 (SPSS Inc., Chicago, IL) was used for the statistical analyses. Categorical variables were expressed as numbers (\%), continuous variables as means ( \pm standard deviation) or median (interquartile range, range). Chi-squared test, Student $t$-test, and MannWhitney test were used for group comparison of particular variables. Pearson or Spearman correlation coefficients were used to determine relationships between testosterone, vitamin D, and characteristics of the study population. To identify factors that contributed to the EDS, binary logistic regression was used. Stepwise multiple linear regression analysis was used to identify factors that contributed to testosterone and vitamin D. All tests were 2 -sided. $\mathrm{p}<0.05$ were considered statistically significant.

The study was approved by the institutional ethics committee and all patients provided an informed consent.

\section{Results}

Our study population consisted of 291 male habitual snorers with the mean age 53.4 \pm 13.1 . Sleep apnea was present in $265(91.1 \%)$ and EDS in $83(28.5 \%)$ of the subjects. Median ESS was 6 (range: $0-24)$. Decreased testosterone levels were present in 41 subjects $(14.1 \%)$ and insufficient vitamin D levels in 231 subjects $(79.4 \%)$. Other characteristics are presented in Table 1. Subjects with EDS had significantly higher use of antihistamines ( $8.4 \%$ vs. $2.4 \%)$, significantly higher frequency of sleep apnea (97.6\% vs. $88.5 \%$ ), significantly higher values of multiple SDB-specific indices, and significantly higher rate of decreased testosterone levels $(20.5 \%$ vs. $11.5 \%)$, compared to subjects without EDS (Table 1). The only variables significantly associated with EDS in binary logistic regression analysis were RDI (95\% CI: 1.0041.024, $\mathrm{p}=0.005$ ) and use of antihistamines (95\% CI: 1.083-11.901, $\mathrm{p}=0.037)$. Characteristics of 265 sleep apnea subjects (with $\mathrm{AHI} \geq 5$ ) are shown in Table 2. Similarly to previous findings, sleep apnea subjects with EDS had significantly higher use of antihistamines (8.6\% vs. $2.7 \%$ ) and RDI (median 46.1 vs. median 34.8), Table 2). Among sleep apnea subjects, RDI (95\% CI: 1.001-1.022, $\mathrm{p}=0.033)$ and use of antihistamines (95\% CI: 1.134-13.317, $\mathrm{p}=0.031)$ were the only independent variables significantly associated with EDS in binary logistic regression analysis. Characteristics of 85 apparently healthy subjects are shown in Table 3 . In subpopulation of apparently healthy subjects, snorers with EDS had significantly higher AI (median 18.6 vs. median 12.4), (Table 3). Among apparently healthy subjects, AI (95\% CI: 1.004-1.060, p=0.027) was the only independent variable significantly associated with EDS in binary logistic regression analysis.

There were significant negative correlations between testosterone levels and both, BMI and nocturnal saturation of blood with oxygen, and significant positive correlations between testosterone and the presence of SDB, AHI, RDI, ODI, and AI (Table 4). BMI (Beta= $-0.282, \mathrm{p}<0.001)$ and ODI $($ Beta $=-0.150, \mathrm{p}=0.043)$ were the only independent variables significantly associated with the levels of testosterone in linear multiple regression analysis.

There was no significant correlation between vitamin D levels and sleep-related indices (Table 5). We found a significant negative correlation between vitamin D levels and BMI, and BMI (Beta=-0.142, $\mathrm{p}=0.016$ ) was the only independent variable that was significantly associated with vitamin D levels in linear multiple regression analysis.

\section{Discussion}

Despite a high frequency of vitamin D insufficiency $(79.4 \%$ ), and relatively high frequency of decreased testosterone levels (14.1\%), we failed to find any independent association of either testosterone or vitamin D with EDS in habitual snorers. Our results suggest that testosterone and vitamin D deficiency do not seem to play important role in EDS among subjects with SDB. Further results of our study suggest an independent association between severity of SDB and testosterone levels. BMI $($ Beta $=-0.282, \quad \mathrm{p}<0.001)$ and ODI (Beta=-0.150, $\mathrm{p}=0.043$ ) were the only independent variables that were significantly associated with testosterone levels in linear multiple regression analysis. We failed to find any significant association of vitamin D levels with sleep-related indices. BMI (Beta=-0.142, $\mathrm{p}=0.016$ ) was the only independent variable that was significantly associated with vitamin D levels in the regression analysis.

Despite limited data on testosterone status in general Slovak population, decreased testosterone levels $(<10 \mathrm{nmol} / \mathrm{l})$ were observed in $40.2 \%$ of males with abdominal obesity (Fillo et al. 2012). Despite similar populations, prevalence of testosterone deficiency was even higher when compared to our study (40.2\% vs. $14.1 \%)$. This finding may suggest important link between obesity and testosterone deficiency. This is in accordance with our results, where inverse association of testosterone with BMI was found. 
Table 1. Characteristics in the population with excessive daytime sleepiness and in the population without excessive daytime sleepiness.

\begin{tabular}{|c|c|c|c|c|}
\hline & \multirow{2}{*}{ All subjects } & \multicolumn{2}{|c|}{ Excessive daytime sleepiness } & \multirow{2}{*}{$\mathbf{p}$} \\
\hline & & Present & Absent & \\
\hline$N$ & 291 & 83 & 208 & \\
\hline Age (years) & $53.4 \pm 13.1$ & $53.5 \pm 12.4$ & $53.3 \pm 13.4$ & 0.942 \\
\hline Body mass index $\left(\mathrm{kg} / \mathrm{m}^{2}\right)$ & $32.2 \pm 6.2$ & $32.6 \pm 6.4$ & $32.1 \pm 6.1$ & 0.502 \\
\hline Hypertension & $162(55.7 \%)$ & $43(51.8 \%)$ & $119(57.2 \%)$ & 0.402 \\
\hline Diabetes mellitus & $43(14.8 \%)$ & $17(20.5 \%)$ & $26(12.5 \%)$ & 0.083 \\
\hline Coronary artery disease & $27(9.3 \%)$ & $8(9.6 \%)$ & $19(9.1 \%)$ & 0.894 \\
\hline Cancer & $7(2.4 \%)$ & $1(1.2 \%)$ & $6(2.9 \%)$ & 0.398 \\
\hline Hypothyroidism & $13(4.5 \%)$ & $5(6.0 \%)$ & $8(3.8 \%)$ & 0.417 \\
\hline Liver dysfunction & $26(8.9 \%)$ & $11(13.3 \%)$ & $15(7.2 \%)$ & 0.103 \\
\hline Gastrointestinal disturbances & $38(13.1 \%)$ & $10(12.0 \%)$ & $28(13.5 \%)$ & 0.747 \\
\hline Epilepsy & $8(2.7 \%)$ & $0(0 \%)$ & $8(3.8 \%)$ & 0.070 \\
\hline Parkinsonism & $3(1 \%)$ & $1(1.2 \%)$ & $2(1.0 \%)$ & 0.853 \\
\hline Depression & $25(8.6 \%)$ & $9(10.8 \%)$ & $16(7.7 \%)$ & 0.386 \\
\hline Alpha-adrenergic blockers & $35(12 \%)$ & $6(7.2 \%)$ & $29(13.9 \%)$ & 0.112 \\
\hline Beta-blockers & $93(32 \%)$ & $25(30.1 \%)$ & $68(32.7 \%)$ & 0.671 \\
\hline Antihistamines & $12(4.1 \%)$ & $7(8.4 \%)$ & $5(2.4 \%)$ & $0.020 *$ \\
\hline Anxiolytics & $9(3.1 \%)$ & $1(1.2 \%)$ & $8(3.8 \%)$ & 0.240 \\
\hline Total sleep time (min) & $410.9 \pm 45.0$ & $415.3 \pm 41.6$ & $409.2 \pm 46.3$ & 0.292 \\
\hline$A H I(n / h)$ & $30.0,43.7(0.1-107.3)$ & $38.0,45.7(0.4-101.1)$ & $26.7,39.3(0.1-107.3)$ & $0.003 * *$ \\
\hline Sleep apnea $(A H I \geq 5)$ & $265(91.1 \%)$ & $81(97.6 \%)$ & $184(88.5 \%)$ & $0.014 *$ \\
\hline Obstructive sleep apnea & $237(89.4 \%)$ & $73(90.1 \%)$ & $164(89.1 \%)$ & 0.809 \\
\hline Central sleep apnea & $28(10.6 \%)$ & $8(9.9 \%)$ & $20(10.9 \%)$ & 0.809 \\
\hline$R D I(n / h)$ & $33.0,42.4(0.4-108.0)$ & $42.5,43.9(3.0-103.9)$ & $30.2,41.7(0.4-108.0)$ & $0.002 * *$ \\
\hline$O D I(n / h)$ & $26.3,39.2(0.1-135.5)$ & $36.3,46.7(0.1-113.0)$ & $23.0,33.7(0.8-135.5)$ & $0.005^{* *}$ \\
\hline Average $\mathrm{SaO}_{2}(\%)$ & $87.8 \pm 6.5$ & $87.2 \pm 6.7$ & $88.0 \pm 6.5$ & 0.332 \\
\hline Minimal $\mathrm{SaO}_{2}(\%)$ & $77.0 \pm 13.5$ & $74.2 \pm 15.6$ & $78.1 \pm 12.5$ & $0.026^{*}$ \\
\hline$A I(n / h)$ & $16.8,23.0(2.6-108.0)$ & $24.3,24.8(4.4-75.0)$ & $15.9,22.3(2.6-108.0)$ & $0.004 * *$ \\
\hline$R L S$ & $43(14.8 \%)$ & $16(19.3 \%)$ & $27(13.0 \%)$ & 0.172 \\
\hline ESS & $6.0,6.0(0-24.0)$ & $13.0,4.0(10.0-24.0)$ & $5.0,4.0(0-9)$ & $<0.001 * * *$ \\
\hline Testosterone (nmol/l) & $13.5 \pm 6.0$ & $13.2 \pm 6.2$ & $13.7 \pm 5.9$ & 0.478 \\
\hline Decreased testosterone (\%) & $41(14.1 \%)$ & $17(20.5 \%)$ & $24(11.5 \%)$ & $0.048^{*}$ \\
\hline$D$ vitamin $(\mu g / l)$ & $22.7 \pm 9.8$ & $21.8 \pm 9.7$ & $23.1 \pm 9.8$ & 0.310 \\
\hline Hypovitaminosis D (\%) & $122(41.9 \%)$ & $47(56.6 \%)$ & $122(58.7 \%)$ & 0.752 \\
\hline Insufficient vitamin D (\%) & $231(79.4 \%)$ & $68(81.9 \%)$ & $163(78.4 \%)$ & 0.498 \\
\hline
\end{tabular}

SDB: sleep-disordered breathing, EDS: excessive daytime sleepiness, AI: arousal index, RDI: respiratory disturbance index, ESS: Epworth sleepiness scale, AHI: apnea-hypopnea index, ODI: oxygen desaturation index, $\mathrm{SaO}_{2}$ : saturation of blood with oxygen, $\mathrm{RLS}$ : restless leg syndrome, $*=p<0.05, * *=p<0.01, * * *=p<0.001$. Decreased testosterone $<9 \mathrm{nmol} / \mathrm{l}$ in $18-50$-year-old; $<7 \mathrm{nmol} / \mathrm{l}$ in $>50$-year-old. Hypovitaminosis $\mathrm{D}<20 \mu \mathrm{g} / \mathrm{l}$. Insufficient vitamin $\mathrm{D}<30 \mu \mathrm{g} / \mathrm{l}$. Categorical variable are expressed as numbers and proportions (\%), continuous variables as means \pm standard deviation or median, interquartile range, minimal-maximal values.

Most of the studies so far studied the association of testosterone with SDB, and data regarding the association of testosterone with other sleep disorders are limited (Wittert 2014). Low testosterone levels in SDB patients have been attributed to hypoxia, sleep deprivation, sleep fragmentation or obesity (Feldman et al. 2002, Luboshitzky et al. 2003, Schmid et al. 2012). On the other hand, the causal role of SDB in decreased testosterone levels remains controversial and the evidence in humans is mixed. While some authors have found an independent association of testosterone with some measures of SDB, other researchers have concluded that 
the low testosterone levels in men with SDB were primarily related to obesity (Clarke et al. 2020, Bercea et al. 2015, Canguven et al. 2010, Luboshitzky et al. 2005). In a recent study, Clarke et al. (2020) suppose, that testosterone concentration is determined by obesity, rather than by sleep apnea per se. This statement is supported by graded effect of weight loss, but limited effect of continuous positive airway pressure therapy to increase testosterone levels and highlights the importance of obesity in men with low testosterone levels.

Table 2. Characteristics in the sleep apnea subjects (apnea-hypopnea index $\geq 5$ ) with excessive daytime sleepiness and without excessive daytime sleepiness.

\begin{tabular}{|c|c|c|c|c|}
\hline & \multirow{2}{*}{ All subjects } & \multicolumn{2}{|c|}{ Excessive daytime sleepiness } & \multirow{2}{*}{$\mathbf{P}$} \\
\hline & & Present & Absent & \\
\hline$N$ & 265 & 81 & 184 & \\
\hline Age (years) & $54.4 \pm 12.3$ & $54.0 \pm 12.1$ & $54.6 \pm 12.4$ & 0.714 \\
\hline Body mass index $\left(\mathrm{kg} / \mathrm{m}^{2}\right)$ & $32.7 \pm 6.1$ & $32.8 \pm 6.4$ & $32.7 \pm 6.0$ & 0.965 \\
\hline Hypertension & $156(58.9 \%)$ & $43(53.1 \%)$ & $113(61.4 \%)$ & 0.204 \\
\hline Diabetes mellitus & $43(16.2 \%)$ & $17(21.0 \%)$ & $26(14.1 \%)$ & 0.163 \\
\hline Coronary artery disease & $27(10.2 \%)$ & $8(9.9 \%)$ & $19(10.3 \%)$ & 0.911 \\
\hline Cancer & $7(2.6 \%)$ & $1(1.2 \%)$ & $6(3.3 \%)$ & 0.343 \\
\hline Hypothyroidism & $11(4.2 \%)$ & $5(6.2 \%)$ & $6(3.3 \%)$ & 0.274 \\
\hline Liver dysfunction & $25(9.4 \%)$ & $11(13.6 \%)$ & $14(7.6 \%)$ & 0.125 \\
\hline Gastrointestinal disturbances & $37(14.0 \%)$ & $10(12.3 \%)$ & $27(14.7 \%)$ & 0.614 \\
\hline Epilepsy & $3(1.1 \%)$ & $0(0 \%)$ & $3(1.6 \%)$ & 0.248 \\
\hline Parkinsonism & $3(1.1 \%)$ & $1(1.2 \%)$ & $2(1.1 \%)$ & 0.917 \\
\hline Depression & $24(9.1 \%)$ & $9(11.1 \%)$ & $15(8.2 \%)$ & 0.439 \\
\hline Alpha-adrenergic blockers & $33(12.5 \%)$ & $6(7.4 \%)$ & $27(14.7 \%)$ & 0.099 \\
\hline Beta-blockers & $93(35.1 \%)$ & $25(30.9 \%)$ & $68(37.0 \%)$ & 0.338 \\
\hline Antihistamines & $12(4.5 \%)$ & $7(8.6 \%)$ & $5(2.7 \%)$ & $0.033^{*}$ \\
\hline Anxiolytics & $7(2.6 \%)$ & $1(1.2 \%)$ & $6(3.3 \%)$ & 0.343 \\
\hline Total sleep time (min) & $413.5 \pm 44.3$ & $415.3 \pm 42.1$ & $412.6 \pm 45.3$ & 0.646 \\
\hline$A H I(n / h)$ & $32.3,43.6(5.2-107.3)$ & $39.3,44.3(6.3-101.1)$ & $31.0,41.7(5.2-107.3)$ & 0.051 \\
\hline \multicolumn{5}{|l|}{ Sleep apnea } \\
\hline Obstructive sleep apnea & $237(89.4 \%)$ & $73(90.1 \%)$ & $164(89.1 \%)$ & 0.809 \\
\hline Central sleep apnea & $28(10.6 \%)$ & $8(9.9 \%)$ & $20(10.9 \%)$ & 0.809 \\
\hline$R D I(n / h)$ & $35.6,42.2(6.1-108.0)$ & $46.1,43.4(8.4-103.9)$ & $34.8,40.8(6.1-108.0)$ & $0.031 *$ \\
\hline$O D I(n / h)$ & $30.3,41.4(1.8-135.5)$ & $37.6,45.9(3.6-113.0)$ & $27.0,37.4(1.8-135.5)$ & 0.096 \\
\hline Average $\mathrm{SaO}_{2}(\%)$ & $87.2 \pm 6.6$ & $87.0 \pm 6.7$ & $87.4 \pm 6.6$ & 0.682 \\
\hline Minimal $\mathrm{SaO}_{2}(\%)$ & $75.7 \pm 13.5$ & $73.8 \pm 15.6$ & $76.6 \pm 12.4$ & 0.123 \\
\hline$A I(n / h)$ & $18.3,25.5(3.5-108.0)$ & $24.3,24.8(4.4-72.3)$ & $16.8,24.2(3.5-108.0)$ & 0.145 \\
\hline$R L S$ & $41(15.5 \%)$ & $16(19.8 \%)$ & $25(13.6 \%)$ & 0.201 \\
\hline ESS & $7.0,6.0(0-24.0)$ & $13.0,4.0(10.0-24.0)$ & $5.0,4.0(0-9)$ & $<0.001 * * *$ \\
\hline Testosterone (nmol/l) & $13.3 \pm 5.9$ & $13.1 \pm 6.2$ & $13.3 \pm 5.8$ & 0.748 \\
\hline Decreased testosterone (\%) & $40(15.1 \%)$ & $17(21.0 \%)$ & $23(12.5 \%)$ & 0.075 \\
\hline$D$ vitamin $(\mu g / l)$ & $22.7 \pm 9.9$ & $21.9 \pm 9.8$ & $23.1 \pm 9.9$ & 0.370 \\
\hline Hypovitaminosis D (\%) & $113(42.6 \%)$ & $35(43.2 \%)$ & $78(42.4 \%)$ & 0.901 \\
\hline Insufficient vitamin D (\%) & $209(78.9 \%)$ & $66(81.5 \%)$ & $143(77.7 \%)$ & 0.489 \\
\hline
\end{tabular}

SDB: sleep-disordered breathing, EDS: excessive daytime sleepiness, AI: arousal index, RDI: respiratory disturbance index, ESS: Epworth sleepiness scale, AHI: apnea-hypopnea index, ODI: oxygen desaturation index, $\mathrm{SaO}_{2}$ : saturation of blood with oxygen, RLS: restless leg syndrome, $*=p<0.05, * *=p<0.01, * * *=p<0.001$. Decreased testosterone $<9 \mathrm{nmol} / \mathrm{l}$ in $18-50$-year-old; $<7 \mathrm{nmol} / \mathrm{l}$ in $>50$-year-old. Hypovitaminosis $\mathrm{D}<20 \mu \mathrm{g} / \mathrm{l}$. Insufficient vitamin $\mathrm{D}<30 \mu \mathrm{g} / \mathrm{l}$. Categorical variable are expressed as numbers and proportions (\%), continuous variables as means \pm standard deviation or median, interquartile range, minimal-maximal values. 
The biggest strength of our study compared to these earlier studies is our larger sample size. In 291 consecutive male habitual snorers, BMI $($ Beta $=-0.282, p<0.001)$ and ODI $($ Beta $=-0.150, p=0.043)$ were the only independent variables significantly associated with levels of testosterone in linear multiple regression analysis. Our results suggest an independent role of both, obesity and nocturnal desaturations with low testosterone levels. However, the study is not designed to address any causal relationship between SDB and low testosterone levels. To elucidate this relationship, future studies should also focus on the treatment of testosterone deficiency and its impact on sleep characteristics.

Vitamin D deficiency $(\leq 20 \mu \mathrm{g} / \mathrm{ml})$ was observed in $41.9 \%$ and vitamin D insufficiency $(\leq 30 \mu \mathrm{g} / \mathrm{ml})$ in $79.4 \%$ of subjects in this study. Despite the data on vitamin D status in general Slovak population are not available, vitamin D deficiency was observed in $15 \%$, and vitamin D insufficiency in $41 \%$ of apparently healthy volunteers, suggesting higher prevalence of decreased vitamin D levels in subjects with SDB
(Sebekova et al. 2016).

The relationship between sleep disorders and vitamin D seems to be complex and bidirectional (McCarty et al. 2014). In cross-sectional studies, lower serum vitamin D levels were associated with higher odds of short sleep duration $(<5 \mathrm{~h})$, and SDB and short sleep duration were independently associated with the risk of vitamin D deficiency (Massa et al. 2015, Piovezan et al. 2017). Mete et al. found no statistically significant difference in serum vitamin D levels between SDB subjects and controls. However, patients with severe SDB had significantly lower levels of vitamin D compared with other groups (Mete et al. 2013). Serum vitamin D has also been assessed in patients with EDS or RLS. In a consecutive series of 81 sleep clinic patients, McCarthy et al. (2012) found an inverse correlation of ESS with vitamin D levels in subjects without vitamin D deficiency. Data from a study of 36 patients with RLS and 38 healthy controls also suggest a significant inverse correlation between vitamin D levels and disease severity in female subjects (Balaban et al. 2012).

Table 3. Characteristics of apparently healthy snorers in the population with excessive daytime sleepiness and in the population without excessive daytime sleepiness.

\begin{tabular}{|c|c|c|c|c|}
\hline & \multirow{2}{*}{ All subjects } & \multicolumn{2}{|c|}{ Excessive daytime sleepiness } & \multirow{2}{*}{$\mathbf{P}$} \\
\hline & & Present & Absent & \\
\hline$N$ & 85 & 23 & 62 & \\
\hline Age (years) & $43.0 \pm 10.3$ & $44.3 \pm 10.8$ & $42.5 \pm 10.2$ & 0.496 \\
\hline Body mass index $\left(\mathrm{kg} / \mathrm{m}^{2}\right)$ & $29.9 \pm 4.7$ & $29.4 \pm 4.5$ & $30.1 \pm 4.8$ & 0.548 \\
\hline Total sleep time (min) & $405.0 \pm 40.0$ & $406.3 \pm 41.3$ & $404.6 \pm 39.8$ & 0.857 \\
\hline$A H I(n / h)$ & $17.3,38.0(0.4-101.1)$ & $27.6,51.2(0.4-101.1)$ & $15.4,33.7(0.9-100.3)$ & 0.133 \\
\hline Sleep apnea $(A H I \geq 5)$ & $71(83.5 \%)$ & $21(91.3 \%)$ & $50(83.3 \%)$ & 0.239 \\
\hline Obstructive sleep apnea & $65(91.5 \%)$ & $19(90.5 \%)$ & $46(92 \%)$ & 0.833 \\
\hline Central sleep apnea & $6(8.5 \%)$ & $2(9.5 \%)$ & $4(8 \%)$ & 0.833 \\
\hline$R D I(n / h)$ & $21.8,37.1(1.4-101.3)$ & $30.3,29.1(3.0-101.3)$ & 20.0, $31.2(1.4-98.7)$ & 0.115 \\
\hline$O D I(n / h)$ & $15.4,33.8(0.1-110.1)$ & $24.5,39.4(0.1-110.1)$ & $13.8,28.2(0.8-100.8)$ & 0.168 \\
\hline Average $\mathrm{SaO}_{2}(\%)$ & $89.1 \pm 7.0$ & $89.2 \pm 5.4$ & $89.1 \pm 7.5$ & 0.974 \\
\hline Minimal $\mathrm{SaO}_{2}(\%)$ & $81.4 \pm 10.6$ & $79.6 \pm 11.4$ & $82.0 \pm 10.3$ & 0.355 \\
\hline$A I(n / h)$ & $13.0,21.8(2.6-75.0)$ & $18.6,30.1(6.1-75.0)$ & $12.4,15.8(2.6-56.5)$ & $0.025^{*}$ \\
\hline$R L S$ & $9(10.6 \%)$ & $1(4.3 \%)$ & $8(12.9 \%)$ & 0.255 \\
\hline$E S S$ & $7.0,6.0(0-20.0)$ & $13.0,3.0(10.0-20.0)$ & $5.0,4.0(0-9)$ & $<0.001 * * *$ \\
\hline Testosterone (nmol/l) & $15.0 \pm 6.0$ & $15.0 \pm 6.2$ & $15.0 \pm 6.1$ & 0.958 \\
\hline Decreased testosterone (\%) & $12(14.1 \%)$ & $3(13.0 \%)$ & $9(14.5 \%)$ & 0.862 \\
\hline$D$ vitamin $(\mu g / l)$ & $22.7 \pm 9.9$ & $23.5 \pm 9.1$ & $22.4 \pm 10.2$ & 0.675 \\
\hline Hypovitaminosis D (\%) & $39(45.9 \%)$ & $9(39.1 \%)$ & $30(48.4 \%)$ & 0.447 \\
\hline Insufficient vitamin D (\%) & $69(81.2 \%)$ & $19(82.6 \%)$ & $50(80.6 \%)$ & 0.837 \\
\hline
\end{tabular}

SDB: sleep-disordered breathing, EDS: excessive daytime sleepiness, AI: arousal index, RDI: respiratory disturbance index, ESS: Epworth sleepiness scale, AHI: apnea-hypopnea index, ODI: oxygen desaturation index, $\mathrm{SaO}_{2}$ : saturation of blood with oxygen, RLS: restless leg syndrome, ${ }^{*}=p<0.05, * * *=p<0.001$. Decreased testosterone $<9 \mathrm{nmol} / \mathrm{l}$ in $18-50$-year-old; $<7 \mathrm{nmol} / \mathrm{l}$ in $>50$-year-old. Hypovitaminosis $D<20 \mu \mathrm{g} / \mathrm{l}$. Insufficient vitamin $\mathrm{D}<30 \mu \mathrm{g} / \mathrm{l}$. Categorical variable are expressed as numbers and proportions (\%), continuous variables as means \pm standard deviation or median, interquartile range, minimal-maximal values. 
Table 4. Correlations of testosterone with sleep-related indices.

\begin{tabular}{lcc}
\hline & $\mathbf{R}$ & $\mathbf{P}$ \\
\hline Age & -0.065 & 0.268 \\
Body mass index & -0.403 & $<0.001 * * *$ \\
Total sleep time & -0.037 & 0.528 \\
AHI & -0.352 & $<0.001^{* * *}$ \\
Sleep apnea & -0.168 & $0.004^{* *}$ \\
RDI & -0.346 & $<0.001 * * *$ \\
ODI & -0.365 & $<0.001 * * *$ \\
Average $\mathrm{SaO}_{2}$ & 0.370 & $<0.001 * * *$ \\
Minimal $\mathrm{SaO}_{2}$ & 0.384 & $<0.001 * * *$ \\
AI & -0.249 & $<0.001 * * *$ \\
ESS & -0.053 & 0.369 \\
EDS & -0.049 & 0.405 \\
RLS & 0.050 & 0.397 \\
\hline
\end{tabular}

EDS: excessive daytime sleepiness, AI: arousal index, RDI: respiratory disturbance index, ESS: Epworth sleepiness scale, AHI: apneahypopnea index, ODI: oxygen desaturation index, $\mathrm{SaO}_{2}$ : saturation of blood with oxygen, RLS: restless leg syndrome. $* * *=p<0.001$, $* *=p<0.01$.

Table 5. Correlations of vitamin D with sleep-related indices.

\begin{tabular}{lcc}
\hline & $\mathbf{R}$ & $\mathbf{P}$ \\
\hline Age & 0.080 & 0.176 \\
Body mass index & -0.126 & $0.031^{*}$ \\
Total sleep time & -0.010 & 0.870 \\
AHI & -0.025 & 0.676 \\
Sleep apnea & -0.011 & 0.846 \\
RDI & -0.021 & 0.726 \\
ODI & -0.055 & 0.354 \\
Average $\mathrm{SaO}_{2}$ & 0.066 & 0.259 \\
Minimal SaO & 0.065 & 0.267 \\
AI & -0.016 & 0.789 \\
ESS & -0.081 & 0.166 \\
EDS & -0.056 & 0.345 \\
RLS & -0.091 & 0.120 \\
\hline
\end{tabular}

EDS: excessive daytime sleepiness, AI: arousal index, RDI: respiratory disturbance index, ESS: Epworth sleepiness scale, AHI: apneahypopnea index, ODI: oxygen desaturation index, $\mathrm{SaO}_{2}$ : saturation of blood with oxygen, RLS: restless leg syndrome. $* * *=p<0.05$, $* *=p<0.01$.

Multiple mechanisms could link vitamin D deficiency with SDB, including immune dysregulation with subsequent development of chronic rhinitis, tonsillar hypertrophy, and impaired contractility and remodeling of airway smooth muscle (Britt et al. 2016, McCarty et al. 2014). Inadequacy of vitamin D is also associated with other conditions, including nonspecific pain and noninflammatory myopathy (Bischoff et al. 1999,
Plotnikoff and Quigley 2003). These conditions could directly contribute to sleep disruption or, conversely, their symptoms could be negatively impacted by poor sleep quality (McCarty et al. 2014).

Compared to these earlier studies on vitamin D, our study is unique in its prospective design as well as consecutive enrollment of patients during a one-year period. In a group of 291 male habitual snorers, we failed 
to find any significant association of vitamin $\mathrm{D}$ with sleeprelated indices of SDB, EDS and presence of RLS. Our results are in accordance with the findings of recent well designed study, where no association was found between severity of sleep apnea and vitamin D levels (Yassa et al. 2019). Future controlled studies are needed to explore the relationship between vitamin $\mathrm{D}$ and sleep disorders, and effects of vitamin D supplementation on sleep parameters should also be investigated.

The primary aim of this study was to explore the predictors of EDS in habitual snorers who were referred for sleep apnea evaluation. The role of testosterone and vitamin D was studied in detail. Despite increasing data linking vitamin $\mathrm{D}$ insufficiency and decreased testosterone levels with EDS and fatigue, their role in sleep disorders remains controversial (Bercea et al. 2015, McCarty et al. 2014). Fatigue and "lack of energy" have been described in non-SDB subjects with low testosterone level (Bassil 2011, Hyde et al. 2010). Bercea et al. (2015) in a study of 15 SDB patients and 15 controls, found a strong association of SDB-related fatigue with serum testosterone. As previously mentioned, McCarthy et al. (2012) found an inverse correlation of ESS with vitamin $\mathrm{D}$ levels in subjects without vitamin D deficiency. Our study failed to find any significant independent association of testosterone and vitamin D levels with EDS, suggesting minor role of testosterone and vitamin D deficiency in EDS among subjects with SDB. RDI (95 \% CI: 1.004-1.024, $\mathrm{p}=0.005)$ and use of antihistamines (95\% CI: 1.083-11.901, $\mathrm{p}=0.037$ ) were the only variables significantly associated with EDS in binary logistic regression analysis. Almost the same results were found when subpopulation of sleep apnea subjects (with AHI index $\geq 5$ ) was analyzed. Similarly, among apparently healthy snorers, AI $(95 \%$ CI: $1.004-1.060, p=0.027)$ was the only independent variable significantly associated with EDS in binary logistic regression analysis.

The lack of fatigue assessment is one of several limitations of our study. The impact of low testosterone levels on fatigue may be more significant than its effect on daytime sleepiness, and this association should be investigated in future prospective studies (Hossain et al. 2005). Another limitation is a tool used for EDS assessment. Although the ESS is a reliable instrument, its self-rating character belongs to the most important limitations of this study. Objective assessment by The Multiple Sleep Latency Test, the gold standard for measuring EDS, is highly warranted in the future studies. Search strategy for EDS predictors also belongs to limitations. Despite the extensive search was performed, we used only the data from the medical records. No additional imaging, functional, or laboratory tests were used. For example, due to absence of thyroid hormones testing, it was not able to clearly assess the control of the hypothyroidism. Future studies should also focus more closely on wider spectrum of comorbid sleep disorders. Insomnia, periodic limb movement disorder, and circadian rhythm disorders were not assessed in this study. In our study, both testosterone and vitamin D levels were inversely associated with BMI. Since BMI might be related to fitness levels, future studies should also assess the impact of physical activity on EDS as well as on testosterone and vitamin $\mathrm{D}$ levels (Basta et al. 2008). Association of antihistamines with sleepiness is also well known, and antihistamines were independently associated with EDS despite their use in only $4.1 \%$ of the subjects in this study (Murri et al. 1992).

Association of sleep disruption with obesity, as well as their adverse consequences are extensively studied (Cibičková et al 2019, Rácz et al. 2018). Regarding the inverse independent association of BMI with both, testosterone and vitamin D levels, future studies should evaluate the role of obesity related hypoventilation in testosterone and vitamin D deficiency. We suppose, that obesity related hypoventilation could play more important role than sleep apnea per se. Due to absence of capnography, we were not able to assess nocturnal hypoventilation. We have to admit one more limitation. Diagnosis of sleep apnea was found in relatively high proportion of enrolled habitual snorers (91.1\%). Enrollment of habitual snorers who were referred for sleep apnea evaluation in sleep laboratory setting could cause selection bias, so prevalence of sleep apnea was higher as expected among "real-world" habitual snorers.

Despite high frequency of vitamin D and testosterone insufficiency, our study failed to find any independent association of testosterone and vitamin $\mathrm{D}$ with EDS in patients with habitual snoring. Our results suggest, that testosterone and vitamin D deficiency do not seem to play important role in EDS in subjects with SDB. No association was found between vitamin D levels and any of the observed sleep-related parameters. While an independent association was found between testosterone levels and the magnitude of nocturnal desaturation, the study is not designed to address any causality in this relationship. Future prospective randomized studies should elucidate any impact of SDB therapy on testosterone and vitamin $\mathrm{D}$ levels. The effects of testosterone and vitamin $\mathrm{D}$ supplementation on sleep parameters should be also investigated. 


\section{Conflict of Interest}

There is no conflict of interest.

\section{Acknowledgements}

This research was supported by the grant APVV-15-0228 and by the Grant of the Framework Program for Research and Technological Development, Project: Completion of the Center of Excellence in Sudden Cerebrovascular Accidents, Faculty of Medicine, Comenius University in Bratislava (ITMS: 26240120023), co-financed by the
European Regional Development Fund.

\section{Abbreviations}

AHI - apnea-hypopnea index, AI - arousal index, BMI body mass index, EDS - excessive daytime sleepiness, ESS - Epworth Sleepiness Scale, ODI - oxygen desaturation index, RDI - respiratory disturbance index, RERAs - respiratory effort related arousals, RLS restless leg syndrome, SDB - sleep-disordered breathing.

\section{References}

ALLEN RP, PICCHIETTI D, HENING WA, TRENKWALDER C, WALTERS AS, MONTPLAISI J: Restless legs syndrome: diagnostic criteria, special considerations, and epidemiology. A report from the restless legs syndrome diagnosis and epidemiology workshop at the National Institutes of Health. Sleep Med 4: 101-119, 2003. http://doi:10.1016/s1389-9457(03)00010-8

AMERICAN ACADEMY OF SLEEP MEDICINE: The International Classification of Sleep Disorders: Diagnostic and Coding Manual. American Academy of Sleep Medicine, Darien, 2014, 383 p.

BALABAN H, YILDIZ OK, CIL G, SENTURK IA, ERSELCAN T, BOLAYIR E, TOPAKTAS S: Serum 25-hydroxyvitamin D levels in restless legs syndrome patients. Sleep Med 13: 953-957, 2012. http://doi:0.1016/j.sleep.2012.04.009

BASSIL N: Late-onset hypogonadism. Med Clin North Am 95: 507-523, 2011. http://doi:10.1016/j.mcna.2011.03.001

BASTA M, LIN HM, PEJOVIC S, SARRIGIANNIDIS A, BIXLER E, VGONTZAS AN: Lack of regular exercise, depression, and degree of apnea are predictors of excessive daytime sleepiness in patients with sleep apnea: sex differences. J Clin Sleep Med 4: 19-25, 2008. http://doi: 18350958

BERCEA RM, MIHAESCU T, COJOCARU C, BJORVATN B: Fatigue and serum testosterone in obstructive sleep apnea patients. Clin Respir J 9: 342-349, 2015. http://doi:10.1111/crj.12150

BISCHOFF HA, STAHELIN HB, URSCHELER N, EHRSAM R, VONTHEIN R, PERRIG-CHIELLO P, TYNDALL A, THEILER R: Muscle strength in the elderly: its relation to vitamin D metabolites. Arch Phys Med Rehabil 80: 54-58, 1999. http://doi:10.1016/s0003-9993(99)90307-6

BOULOUKAKI I, TSILIGIANNI I, MERMIGKIS C, BONSIGNORE MR, MARKAKIS M, PATAKA A, STEIROPOULOS P, ERMIDOU C, ALEXAKI I, TZANAKIS N, SCHIZA S: Vitamin D deficiency in patients evaluated for obstructive sleep apnea: is it associated with disease severity? Sleep Breath 2020. http://doi:10.1007/s11325-020-02142-w

BRITT RD, THOMPSON MA, FREEMAN MR, STEWART AL, PABELICK CM, PRAKASH YS: Vitamin D reduces inflammation-induced contractility and remodeling of asthmatic human airway smooth muscle. Ann Am Thorac Soc 13 (Suppl 1): S97-S98, 2016. http://doi:10.1513/AnnalsATS.201508-540MG

CANGUVEN O, SALEPCI B, ALBAYRAK S, SELIMOGLU A, BALABAN M, BULBUL M: Is there a correlation between testosterone levels and the severity of the disease in male patients with obstructive sleep apnea? Arch Ital Urol Androl 82: 143-147, 2010.

CIBIČKOVÁ L, LANGOVÁ K, VAVERKOVÁ H, LUKEŠ J, CIBIČEK N, KARÁSEK D: Superior role of waist circumference to body-mass index in the prediction of cardiometabolic risk in dyslipidemic patients. Physiol Res 68: 931-938, 2019. http://doi:10.33549/physiolres.934176

CLARKE BM, VINCENT AD, MARTIN S, ADAMS R, APPLETON S, VAKULIN A, JESUDASON D, WITTERT GA: Obstructive sleep apnea is not an independent determinant of testosterone in men. Eur J Endocrinol 183: 31-39, 2020. http://doi:10.1530/EJE-19-0978

COWIE MR: Sleep apnea: State of the art. Trends Cardiovasc Med 27: 280-289, 2017. http://doi:10.1016/j.tcm.2016.12.005 
FELDMAN HA, LONGCOPE C, DERBY CA, JOHANNES CB, ARAUJO AB, COVIELLO AD, BREMNER WJ, MCKINLAY JB: Age trends in the level of serum testosterone and other hormones in middle-aged men: longitudinal results from the Massachusetts male aging study. J Clin Endocrinol Metab 87: 589-598, 2002. http://doi:10.1210/jcem.87.2.8201

FILLO J, BREZA J, LEVČÍKOVÁ M, LUHA J, VACHULOVÁ A, DURDÍK Š, LABAŠ P: Occurrence of erectile dysfunction, testosterone deficiency syndrome and metabolic syndrome in patients with abdominal obesity. Where is a sufficient level of testosterone? Int Urol Nephrol 44: 1113-1120, 2012. http://doi:10.1007/s11255$\underline{012-0132-1}$

GUILLEMINAULT C, BROOKS SN: Excessive daytime sleepiness: a challenge for the practising neurologist. Brain 124: 1482-1491, 2001. http://doi:10.1093/brain/124.8.1482

HOSSAIN JL, AHMAD P, REINISH LW, KAYUMOV L, HOSSAIN NK, SHAPIRO CM: Subjective fatigue and subjective sleepiness: two independent consequences of sleep disorders? J Sleep Res 14: 245-253, 2005. http://doi:10.1111/j.1365-2869.2005.00466.x

HYDE Z, FLICKER L, ALMEIDA OP, HANKEY GJ, MCCAUL KA, CHUBB SA, YEAP BB: Low free testosterone predicts frailty in older men: the health in men study. J Clin Endocrinol Metab 95: 3165-3172, 2010. http://doi:10.1210/jc.2009-2754

IBER C, ANCOLI-ISRAEL S, CHESSON A, QUAN SF: The AASM Manual for the Scoring of Sleep and Associated Events: Rules, Terminology and Technical Specifications. Westchester: American Academy of Sleep Medicine, 2007, $59 \mathrm{p}$.

JOHNS MW: A new method for measuring daytime sleepiness: The Epworth sleepiness scale. Sleep 14: 540-545, 1991. http://doi:10.1093/sleep/14.6.540

LUBOSHITZKY R, LAVIE L, SHEN-ORR Z, HERER P: Altered luteinizing hormone and testosterone secretion in middle-aged obese men with obstructive sleep apnea. Obes Res 13: 780-786, 2005. http://doi:10.1038/oby.2005.88

LUBOSHITZKY R, SHEN-ORR Z, HERER P: Middle-aged men secrete less testosterone at night than young healthy men. J Clin Endocrinol Metab 88: 3160-3166, 2003. http://doi:10.1210/jc.2002-021920

MASSA J, STONE KL, WEI EK, HARRISON SL, BARRETT-CONNOR E, LANE NE, PAUDEL M, REDLINE S, ANCOLI-ISRAEL S, ORWOLL E, SCHERNHAMMER E: Vitamin D and actigraphic sleep outcomes in older community-dwelling men: the MrOS sleep study. Sleep 38: 251-257, 2015. http://doi:10.5665/sleep.4408

MCCARTY DE, CHESSON AL, JAIN SK, MARINO AA: The link between vitamin D metabolism and sleep medicine. Sleep Med Rev 18: 311-319, 2014. http://doi:10.1016/j.smrv.2013.07.001

MCCARTY DE, REDDY A, KEIGLEY Q, KIM PY, MARINO AA: Vitamin D, race, and excessive daytime sleepiness. J Clin Sleep Med 8: 693-697, 2012. http://doi:10.5664/jcsm.2266

METE T, YALCIN Y, BERKER D, CIFTCI B, GUVEN SF, TOPALOGLU O, YAVUZ HC, GULER S: Obstructive sleep apnea syndrome and its association with vitamin D deficiency. J Endocrinol Invest 36: 681-685, 2013. http://doi:10.3275/8923

MURRI L, MASSETANI R, KRAUSE M, DRAGONETTI C, IUDICE A: Evaluation of antihistamine-related daytime sleepiness. A double-blind, placebo-controlled study with terfenadine. Allergy 47: 532-534, 1992. http://doi:10.1111/j.1398-9995.1992.tb00678.x

PAGEL JF: Medications that induce sleepiness. In: Sleep: A Comprehensive Handbook. LEE-CHIONG T (ed.), John Wiley \& Sons, Inc., 2006, pp 175-182. http://doi:10.1002/0471751723

PAGEL JF: Excessive daytime sleepiness. Am Fam Physician 79: 391-396, 2009.

PEPPARD PE, YOUNG T, BARNET JH, PALTA M, HAGEN EW, HLA KM: Increased prevalence of sleepdisordered breathing in adults. Am J Epidemiol 177: 1006-1014, 2013. http://doi:10.1093/aje/kws342

PIOVEZAN RD, HIROTSU C, FERES MC, CINTRA FD, ANDERSEN ML, TUFIK S, POYARES D: Obstructive sleep apnea and objective short sleep duration are independently associated with the risk of serum vitamin D deficiency. PLoS One 12: e0180901, 2017. http://doi:10.1371/journal.pone.0180901

PLOTNIKOFF GA, QUIGLEY JM: Prevalence of severe hypovitaminosis D in patients with persistent, nonspecific musculoskeletal pain. Mayo Clin Proc 78: 1463-1470, 2003. http://doi:10.4065/78.12.1463 
RÁCZ B, DUŠKOVÁ M, STÁRKA L, HAINER V, KUNEŠOVÁ M: Links between the circadian rhythm, obesity and the microbiome. Physiol Res 28: 409-420, 2018. http://doi:10.33549/physiolres.934020

SEBEKOVA K, KRIVOSIKOVA Z, GAJDOS M, PODRACKA L: Vitamin D status in apparently healthy medicationfree Slovaks: Association to blood pressure, body mass index, self-reported smoking status and physical activity. Bratisl Lek Listy 117: 702-709. 2016. http://doi:10.4149/BLL 2016 135

SCHMID SM, HALLSCHMID M, JAUCH-CHARA K, LEHNERT H, SCHULTES B: Sleep timing may modulate the effect of sleep loss on testosterone. Clin Endocrinol (Oxf) 77: 749-754, 2012. http://doi:10.1111/j.13652265.2012.04419.x

VIANA A, DAFLON AC, COUTO A, NEVES D, DE ARAUJO-MELO MH, CAPASSO R: Nocturnal hypoxemia is associated with low testosterone levels in overweight males and older men with normal weight. J Clin Sleep Med 13: 1395-1401, 2017. https://doi.org/10.5664/jesm.6832

WITTERT G: The relationship between sleep disorders and testosterone in men. Asian J Androl 16: 262-265, 2014. http://doi:10.4103/1008-682X.122586

YASSA OY, DOMAC SF, KENANGIL G: Serum vitamin D status does not correlate with the severity of obstructive sleep apnea in male adults: A controlled study design with minimized factors influencing serum vitamin D levels. Int J Vitam Nutr Res 20: 1-7, 2019. http://doi:10.1024/0300-9831/a000539 